\title{
Root-MUSIC Based Angle Estimation for MIMO Radar with Unknown Mutual Coupling
}

\author{
Jianfeng Li, Xiaofei Zhang, and Weiyang Chen \\ Department of Electronic Engineering, Nanjing University of Aeronautics \& Astronautics, Nanjing 210016, China \\ Correspondence should be addressed to Xiaofei Zhang; njxnd88@126.com
}

Received 5 August 2013; Revised 18 December 2013; Accepted 19 December 2013; Published 16 January 2014

Academic Editor: Hoi-Shun Lui

Copyright (C) 2014 Jianfeng Li et al. This is an open access article distributed under the Creative Commons Attribution License, which permits unrestricted use, distribution, and reproduction in any medium, provided the original work is properly cited.

\begin{abstract}
Direction of arrival (DOA) estimation problem for multiple-input multiple-output (MIMO) radar with unknown mutual coupling is studied, and an algorithm for the DOA estimation based on root multiple signal classification (MUSIC) is proposed. Firstly, according to the Toeplitz structure of the mutual coupling matrix, output data of some specified sensors are selected to eliminate the influence of the mutual coupling. Then the reduced-dimension transformation is applied to make the computation burden lower as well as obtain a Vandermonde structure of the direction matrix. Finally, Root-MUSIC can be adopted for the angle estimation. The angle estimation performance of the proposed algorithm is better than that of estimation of signal parameters via rotational invariance techniques (ESPRIT)-like algorithm and MUSIC-like algorithm. Furthermore, the proposed algorithm has lower complexity than them. The simulation results verify the effectiveness of the algorithm, and the theoretical estimation error of the algorithm is also derived.
\end{abstract}

\section{Introduction}

Multiple-input multiple-output (MIMO) radars have attracted a lot of attention recently for their potential advantages over conventional phased-array radars [1-4]. MIMO radar systems can overcome fading effect, enhance spatial resolution, strengthen parameter identifiability, and improve target detection performance for the additional degrees of freedom [5-7]. Angle estimation is a key issue in MIMO radar and has been studied by a lot of researchers. Estimation of signal parameters via rotational invariance techniques (ESPRIT) algorithms [8-10] can obtain the closed-form estimations via rotational invariance in the subspace. Capon algorithm [11] and multiple signal classification (MUSIC) algorithm [12] both estimate the angles via the peak searches. Polynomial root finding technique can transform the peak searches into root finding problem when the arrays are uniform linear arrays (ULA) [13]. Based on the uniqueness of trilinear decomposition, parallel factor analysis (PARAFAC) algorithms [14-16] can also be adopted for the angle estimation. For monostatic MIMO radar, low-complexity ESPRIT [17] and Capon [18] can achieve better angle estimation performance with lighter computation burden based on the reduced-dimension transformation. However, these methods strongly depend on the array manifold, which is often perturbed by the mutual coupling in practical situations. The mutual coupling will make the above methods have performance degradation or even fail to work, and various methods for mutual coupling compensation have been studied. The Receiving-Mutual-Impedance Methods (RMIM) [19, 20] can obtain the decoupled voltages via the known terminated impedance and the receiving mode antennas. The calibration methods [21-23], which will be studied in this paper, can utilize the special characteristics of the mutual coupling coefficients of uniform linear arrays (ULA). Reference [24] proposed a MUSIC-like algorithm for the angle estimation in the presence of mutual coupling. Through reconstructing the MUSIC function based on the Toeplitz structure of the mutual coupling matrix, the angles can be estimated via the peak searches. To avoid the high-computational peak search, an ESPRIT-like algorithm was proposed in [25] by removing the auxiliary sensors to eliminate the influence of mutual coupling. The rotational invariance can be extracted in the new data for the angle estimation. 


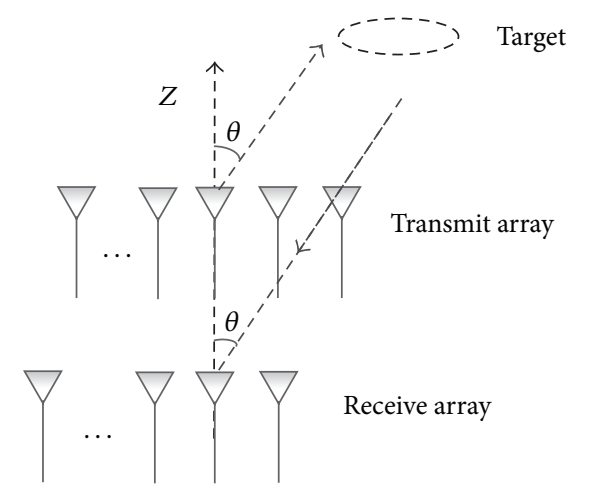

FIGURE 1: Array structure of monostatic MIMO radar.

Although MUSIC-like algorithm can achieve high resolution angle estimation with unknown mutual coupling, the high computational peak searches will make it ineffective. As a faster method, ESPRIT-like can obtain the close-form solution of the angle estimation, but it only exploits the relationship between the subarrays, which will lead to a performance loss.

Our goal is to develop an effective method for angle estimation in MIMO radar with unknown mutual coupling. So, in this paper, we propose a Root-MUSIC based method, which is fast and can deal with the mutual coupling problem, which often occurrs in practical situation. The nonauxiliary sensors are also used to eliminate the influence of mutual coupling. Then a transformation is utilized to reduce the dimension of the data, meanwhile a direction matrix with Vandermonde structure can be obtained. Finally, RootMUSIC, which is a root-finding form of the MUSIC peak searching, can be adopted for the angle estimation. The angle estimation performance of the proposed algorithm is better than that of ESPRIT-like algorithm and MUSIC-like algorithm. Furthermore, it may also have lower complexity.

Notation. $(\cdot)^{T},(\cdot)^{H},(\cdot)^{-1}$, and $(\cdot)^{+}$denote transpose, conjugate-transpose, inverse, and pseudo-inverse operations, respectively. $\operatorname{diag}(\mathbf{v})$ stands for diagonal matrix whose diagonal element is a vector $\mathbf{v} . \mathbf{I}_{K}$ is a $K \times K$ identity matrix. $\otimes$ is the Kronecker product. $\operatorname{Re}(\cdot)$ is to get real part of the complex, and angle $(\cdot)$ means taking phase operation.

\section{Data Model}

As shown in Figure 1, the monostatic MIMO radar system is equipped with both uniformlinear arrays (ULA) for the transmit and receive arrays, and the arrays are both located in the $y$-axis with half-wavelength spacing between adjacent antennas, respectively. Assume that there are $K$ targets in the $y-z$ plane, and the output of the matched filters at the receiver can be expressed as $[5,23,24]$

$$
\mathbf{x}(t)=\left[\widetilde{\mathbf{a}}_{r}\left(\theta_{1}\right) \otimes \widetilde{\mathbf{a}}_{t}\left(\theta_{1}\right), \ldots, \widetilde{\mathbf{a}}_{r}\left(\theta_{K}\right) \otimes \widetilde{\mathbf{a}}_{t}\left(\theta_{K}\right)\right] \mathbf{s}(t)+\mathbf{n}(t),
$$

where $\theta_{K}$ is the direction of arrival (DOA) of the $k$ th target with respect to the transmit array normal or the receive array normal, $\mathbf{s}(t)=\left[s_{1}(t), s_{2}(t), \ldots, s_{K}(t)\right]^{T} \in \mathbb{C}^{K \times 1}, s_{k}(t)=$ $\beta_{k} e^{j 2 \pi f_{k} t}$ with $f_{k}$ and $\beta_{k}$ being the Doppler frequency and the reflect coefficient, respectively, $\mathbf{n}(t)$ is an $M N \times 1$ Gaussian white noise vector of zero mean and covariance matrix $\sigma^{2} \mathbf{I}_{M N}$, and $\widetilde{\mathbf{a}}_{r}\left(\theta_{k}\right)$ and $\widetilde{\mathbf{a}}_{t}\left(\theta_{k}\right)$ are the receive and transmit steering vectors for the $k$ th target under the influence of mutual coupling, respectively. They satisfy

$$
\begin{aligned}
& \widetilde{\mathbf{a}}_{r}\left(\theta_{k}\right)=\mathbf{C}_{r} \mathbf{a}_{r}\left(\theta_{k}\right), \\
& \widetilde{\mathbf{a}}_{t}\left(\theta_{k}\right)=\mathbf{C}_{t} \mathbf{a}_{t}\left(\theta_{k}\right),
\end{aligned}
$$

where $\mathbf{C}_{r} \in \mathbb{C}^{N \times N}$ and $\mathbf{C}_{t} \in \mathbb{C}^{M \times M}$ represent the matrices containing the mutual coupling coefficients. With the assumption of ULA, they are Toeplitz matrices [24]

$$
\begin{aligned}
& \mathbf{C}_{r}=\operatorname{toeplitz}\left(c_{0}^{r}, \ldots, c_{n}^{r}, 0, \ldots, 0\right), \\
& \mathbf{C}_{t}=\operatorname{toeplitz}\left(c_{0}^{t}, \ldots, c_{m}^{t}, 0, \ldots, 0\right),
\end{aligned}
$$

where $c_{p}^{r}(p=0 \ldots n)$ and $c_{q}^{t}(q=0 \ldots m)$ stand for the mutual coupling coefficients. If the first sensors are the reference points, then $c_{0}^{r}=c_{0}^{t}=1 . \mathbf{a}_{r}\left(\theta_{k}\right)$ and $\mathbf{a}_{t}\left(\theta_{k}\right)$ in $(2 \mathrm{a})$ and (2b) are expressed as Vandermonde structures

$$
\begin{aligned}
& \mathbf{a}_{r}\left(\theta_{k}\right)=\left[1, z_{k}, \ldots, z_{k}^{N-1}\right]^{T}, \\
& \mathbf{a}_{t}\left(\theta_{k}\right)=\left[1, z_{k}, \ldots, z_{k}^{M-1}\right]^{T},
\end{aligned}
$$

where $z_{k}=e^{-j \pi \sin \theta_{k}}$.

By collecting $J$ samples, and representing the received data as $\mathbf{X}=[\mathbf{x}(1), \mathbf{x}(2), \ldots, \mathbf{x}(J)]$, the received data can be expressed as

$$
\mathbf{X}=\widetilde{\mathbf{A}} \mathbf{S}+\mathbf{N}
$$

where $\widetilde{\mathbf{A}}=\left[\widetilde{\mathbf{a}}_{r}\left(\theta_{1}\right) \otimes \widetilde{\mathbf{a}}_{t}\left(\theta_{1}\right), \ldots, \widetilde{\mathbf{a}}_{r}\left(\theta_{K}\right) \otimes \widetilde{\mathbf{a}}_{t}\left(\theta_{K}\right)\right] \in \mathbb{C}^{M N \times K}$ is the direction matrix, $\mathbf{S}=[\mathbf{s}(1), \mathbf{s}(2), \ldots, \mathbf{s}(J)] \in \mathbb{C}^{K \times J}$, and $\mathbf{N} \in \mathbb{C}^{M N \times J}$ stands for the noise matrix.

\section{Root-MUSIC Based Angle Estimation for MIMO Radar with Unknown Mutual Coupling}

3.1. Mutual Coupling Elimination. According to [25], define the selecting matrices as

$$
\begin{aligned}
& \mathbf{J}_{1}=\left[\mathbf{0}_{(N-2 n) \times n}, \mathbf{I}_{(N-2 n) \times(N-2 n)}, \mathbf{0}_{(N-2 n) \times n-1}\right], \\
& \mathbf{J}_{2}=\left[\mathbf{0}_{(M-2 m) \times m}, \mathbf{I}_{(M-2 m) \times(M-2 m)}, \mathbf{0}_{(M-2 m) \times m}\right] .
\end{aligned}
$$

Then,

$$
\begin{aligned}
& \mathbf{J}_{1} \widetilde{\mathbf{a}}_{r}\left(\theta_{k}\right)=\mathbf{J}_{1} \mathbf{C}_{r} \mathbf{a}_{r}\left(\theta_{k}\right)=\alpha_{r k} \mathbf{a}_{r 1}\left(\theta_{k}\right), \\
& \mathbf{J}_{2} \widetilde{\mathbf{a}}_{t}\left(\theta_{k}\right)=\mathbf{J}_{2} \mathbf{C}_{t} \mathbf{a}_{t}\left(\theta_{k}\right)=\alpha_{t k} \mathbf{a}_{t 1}\left(\theta_{k}\right),
\end{aligned}
$$

where $\alpha_{r k}=\sum_{p=-n}^{n} c_{|p|}^{r} z_{k}^{p+n}, \alpha_{t k}=\sum_{q=-m}^{m} c_{|q|}^{t} z_{k}^{q+m}$, and $\mathbf{a}_{r 1}\left(\theta_{k}\right)$ and $\mathbf{a}_{t 1}\left(\theta_{k}\right)$ are the first $(N-2 n)$ and $(M-2 m)$ elements of 
$\mathbf{a}_{r}\left(\theta_{k}\right)$ and $\mathbf{a}_{t}\left(\theta_{k}\right)$, respectively. Define $\bar{M}=M-2 m$ and $\bar{N}=$ $N-2 n$, then

$$
\begin{aligned}
& \mathbf{a}_{r 1}\left(\theta_{k}\right)=\left[1, z_{k}, \ldots, z_{k}^{\bar{N}-1}\right]^{T}, \\
& \mathbf{a}_{t 1}\left(\theta_{k}\right)=\left[1, z_{k}, \ldots, z_{k}^{\bar{M}-1}\right]^{T} .
\end{aligned}
$$

The new steering vector of the output can be now characterized as

$$
\begin{aligned}
\mathbf{a}_{c}\left(\theta_{k}\right) & =\left(\mathbf{J}_{1} \otimes \mathbf{J}_{2}\right)\left(\widetilde{\mathbf{a}}_{r}\left(\theta_{k}\right) \otimes \widetilde{\mathbf{a}}_{t}\left(\theta_{k}\right)\right) \\
& =\alpha_{r k} \alpha_{t k} \mathbf{a}_{r 1}\left(\theta_{k}\right) \otimes \mathbf{a}_{t 1}\left(\theta_{k}\right) .
\end{aligned}
$$

According to (9), the steering vector can be expressed as a kronecker product of two Vandermonde vectors without considering the scalar coefficients $\alpha_{r k}$ and $\alpha_{t k}$. Thus, the mutual coupling can be considered to be eliminated through this procedure.

3.2. Reduced-Dimension Transformation. The length of the new steering vector $\mathbf{a}_{c}\left(\theta_{k}\right)$ is $\overline{M N}$, which is too long and will add high computation burden. Furthermore, it will make the later root finding hard to implement for the high-order polynomials. So the reduced-dimension transformation is necessary. According to [17], steering vectors with Vandermonde structures will satisfy

$$
\mathbf{a}_{r 1}\left(\theta_{k}\right) \otimes \mathbf{a}_{t 1}\left(\theta_{k}\right)=\mathbf{G b}\left(\theta_{k}\right),
$$

where

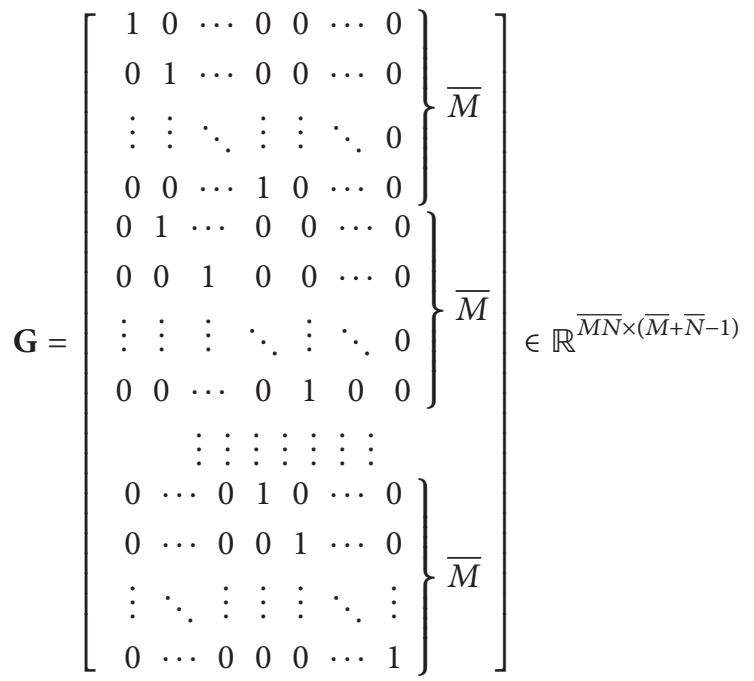

$\mathbf{b}\left(\theta_{k}\right)=\left[1, z_{k}, \ldots, z_{k}^{\bar{M}+\bar{N}-2}\right]^{T} \in \mathbb{C}^{(\bar{M}+\bar{N}-1) \times 1}$. Then define $\mathbf{W} \triangleq$ $\mathbf{G}^{H} \mathbf{G}$,

$$
=\operatorname{diag}(1,2, \ldots, \underbrace{\min (\bar{M}, \bar{N}), \ldots, \min (\bar{M}, \bar{N})}_{|\bar{M}-\bar{N}|+1}, \ldots, 2,1) .
$$

Using the reduced-dimension transformation $\mathbf{W}^{-1 / 2} \mathbf{G}^{H}$ for the receive signal after the mutual coupling elimination, and combining (9) and (10), then

$$
\begin{aligned}
\mathbf{y}(t)= & \mathbf{W}^{-1 / 2} \mathbf{G}^{H}\left(\mathbf{J}_{1} \otimes \mathbf{J}_{2}\right) \mathbf{x}(t) \\
= & \mathbf{W}^{-1 / 2} \mathbf{G}^{H}\left[\mathbf{a}_{c}\left(\theta_{1}\right), \mathbf{a}_{c}\left(\theta_{2}\right), \ldots, \mathbf{a}_{c}\left(\theta_{K}\right)\right] \mathbf{s}(t) \\
& +\mathbf{W}^{-1 / 2} \mathbf{G}^{H}\left(\mathbf{J}_{1} \otimes \mathbf{J}_{2}\right) \mathbf{n}(t) \\
= & \mathbf{W}^{-1 / 2} \mathbf{W}\left[\mathbf{b}\left(\theta_{1}\right), \mathbf{b}\left(\theta_{2}\right), \ldots, \mathbf{b}\left(\theta_{K}\right)\right] \mathbf{\Phi} \mathbf{s}(t)+\mathbf{n}_{y}(t) \\
= & \mathbf{W}^{1 / 2} \mathbf{B} \Phi \mathbf{s}(t)+\mathbf{n}_{y}(t),
\end{aligned}
$$

where $\mathbf{B}=\left[\mathbf{b}\left(\theta_{1}\right), \mathbf{b}\left(\theta_{2}\right), \ldots, \mathbf{b}\left(\theta_{K}\right)\right], \boldsymbol{\Phi}=\operatorname{diag}\left(\alpha_{r 1} \alpha_{t 1}, \ldots\right.$, $\left.\alpha_{r K} \alpha_{t K}\right)$ and $\mathbf{n}_{y}(t)=\mathbf{W}^{-1 / 2} \mathbf{G}^{H}\left(\mathbf{J}_{1} \otimes \mathbf{J}_{2}\right) \mathbf{n}(t)$. The covariance matrix of $\mathbf{y}(t)$ is

$$
\begin{aligned}
\mathbf{R}= & E\left[\mathbf{y}(t) \mathbf{y}^{H}(t)\right] \\
= & \mathbf{W}^{1 / 2} \mathbf{B} \boldsymbol{\Phi}\left(E\left[\mathbf{s}(t) \mathbf{s}^{H}(t)\right]\right) \mathbf{\Phi}^{H} \mathbf{B}^{H} \mathbf{W}^{1 / 2} \\
& +E\left[\mathbf{n}_{y}(t) \mathbf{n}_{y}^{H}(t)\right] \\
= & \mathbf{W}^{1 / 2} \mathbf{B R}_{s} \mathbf{B}^{H} \mathbf{W}^{1 / 2}+\sigma^{2} \mathbf{I}_{L},
\end{aligned}
$$

where $\mathbf{R}_{s}=\boldsymbol{\Phi}\left(E\left[\mathbf{s}(t) \mathbf{s}^{H}(t)\right]\right) \boldsymbol{\Phi}^{H}$ and $L=\bar{M}+\bar{N}-1 . \mathbf{W}^{1 / 2} \mathbf{B}$ can be regarded as the new direction matrix.

3.3. Root-MUSIC Based Angle Estimation. The covariance matrix in (14) can be decomposed as

$$
\mathbf{R}=\mathbf{E}_{s} \mathbf{D}_{s} \mathbf{E}_{s}^{H}+\mathbf{E}_{n} \mathbf{D}_{n} \mathbf{E}_{n}^{H},
$$

where $\mathbf{D}_{s}$ denotes a $K \times K$ diagonal matrix formed by $K$ largest eigenvalues $\lambda_{1}, \ldots, \lambda_{K}$ and $\mathbf{D}_{n}$ denotes a diagonal matrix formed by the rest $L-K$ smaller eigen-values $\left(\lambda_{K+1}=\lambda_{K+2}=\right.$ $\left.\cdots=\lambda_{L}=\sigma^{2}\right) . \mathbf{E}_{s}$ and $\mathbf{E}_{n}$ represent the signal subspace and noise subspace, respectively, of which $\mathbf{E}_{s}$ stands for the eigenvectors corresponding to the $K$ largest eigen-values, and $\mathbf{E}_{n}$ consists of the rest eigenvectors.

According to the MUSIC peak search function [12], the space spanned by the direction matrix is orthogonal to the noise subspace, and the angle can be estimated by searching the following function:

$$
F(\theta)=\frac{1}{\mathbf{b}^{H}(\theta) \mathbf{W}^{1 / 2} \mathbf{E}_{n} \mathbf{E}_{n}^{H} \mathbf{W}^{1 / 2} \mathbf{b}(\theta)},
$$

where $\mathbf{b}(\theta)=\left[1, e^{-j \pi \sin \theta}, \ldots, e^{-j(L-1) \pi \sin \theta}\right]^{T}$. To avoid the peak searching, let $z=e^{-j \pi \sin \theta}$; then $\mathbf{b}(\theta)$ can be expressed as $\mathbf{b}(z)=\left[1, z, \ldots, z^{L-1}\right]^{T}$. Equation (16) can be transformed into the root finding problem

$$
\mathbf{b}^{T}\left(\frac{1}{z}\right) \mathbf{U}_{n} \mathbf{b}(z)=0,
$$

where $\mathbf{U}_{n}=\mathbf{W}^{1 / 2} \mathbf{E}_{n} \mathbf{E}_{n}^{H} \mathbf{W}^{1 / 2}$. According to [26], the polynomial in (17) can be expressed as

$$
\left[z^{-L+1}, \ldots, z^{-1}, 1, z, \ldots, z^{L-1}\right] \mathbf{c}=0,
$$


where $\mathbf{c} \in \mathbb{C}^{2 L-1}$ is a column vector which contains the coefficients of the polynomial and the $l$ th coefficient $(\mathbf{c}(l), l=$ $1, \ldots, 2 L-1)$ is given by the sum of the $(l-L)$ th diagonal elements of $\mathbf{U}_{n}$.

The $K$ roots which are most close to the unit circle will be the solutions, and they are denoted as $\widehat{z}_{1}, \ldots, \widehat{z}_{K}$. The angle $\theta_{k}$ can be estimated via

$$
\theta_{k}=-\frac{\operatorname{angle}\left(\widehat{z}_{k}\right)}{\pi}, \quad k=1, \ldots, K .
$$

With respect to (14), the covariance matrix can be estimated via

$$
\widehat{\mathbf{R}}=\frac{1}{J} \sum_{t=1}^{J} \mathbf{y}(t) \mathbf{y}^{H}(t) .
$$

The major steps of the proposed algorithm for DOA estimation in MIMO-radar are shown as follows.

(1) Construct the new output via (13) to eliminate the mutual coupling and reduce the data dimension.

(2) Estimate the covariance matrix of the data through $\widehat{\mathbf{R}}=(1 / J) \sum_{t=1}^{J} \mathbf{y}(t) \mathbf{y}^{H}(t)$.

(3) Perform eigen-value decomposition of $\widehat{\mathbf{R}}$ to get the noise subspace $\widehat{\mathbf{E}}_{n}$.

(4) Construct the MUSIC function and use root finding technique to obtain the angle estimation $\widehat{\theta}_{k}$ via (18)(19).

3.4. Complexity Analysis. The proposed algorithm has lower complexity than ESPRIT-like algorithm and MUSIC-like algorithm for it requires no peak searches and has lower dimension. Figure 2 shows the run time of the three algorithms in computer versus the number of antennas, and here we choose $M=N$ for simplify. From Figure 2, we find that our algorithm has lower complexity, and the change trend versus the number of antennas of the proposed algorithm is smaller than those of the other two algorithms.

\section{Theoretical Analysis and Cramer-Rao Bound (CRB)}

4.1. Theoretical Analysis of the Proposed Algorithm. The decomposition of $\widehat{\mathbf{R}}$ in (20) can be expressed as

$$
\widehat{\mathbf{R}}=\widehat{\mathbf{E}}_{s} \widehat{\mathbf{D}}_{s} \widehat{\mathbf{E}}_{s}^{H}+\widehat{\mathbf{E}}_{n} \widehat{\mathbf{D}}_{n} \widehat{\mathbf{E}}_{n}^{H},
$$

where $\widehat{\mathbf{E}}_{s}$ and $\widehat{\mathbf{E}}_{n}$ denote the estimated signal and noise subspaces, respectively.

According to [27], Root-MUSIC and MUSIC peak search method have the same angle estimation error as they both seek for the extreme points of $f(\theta)=\mathbf{b}^{H}(\theta) \widehat{\mathbf{U}}_{n} \mathbf{b}(\theta)$, where

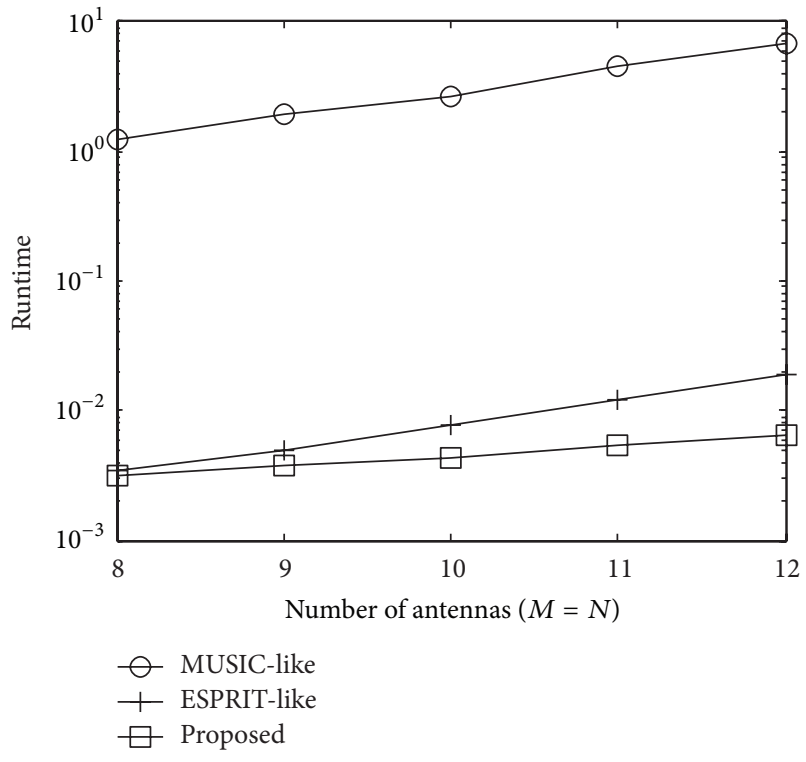

FIGURE 2: Complexity comparison against the number of antennas $(J=100, K=3)$.

$\widehat{\mathbf{U}}_{n}=\mathbf{W}^{1 / 2} \widehat{\mathbf{E}}_{n} \widehat{\mathbf{E}}_{n}^{H} \mathbf{W}^{1 / 2}$. The first-order derivative of $f(\theta)$ on $\widehat{\theta}_{k}$ will be equal to zero; that is,

$$
\begin{aligned}
f^{\prime}\left(\widehat{\theta}_{k}\right) & =\mathbf{d}^{H}\left(\widehat{\theta}_{k}\right) \widehat{\mathbf{U}}_{n} \mathbf{b}\left(\widehat{\theta}_{k}\right)+\mathbf{b}^{H}\left(\widehat{\theta}_{k}\right) \widehat{\mathbf{U}}_{n} \mathbf{d}\left(\widehat{\theta}_{k}\right) \\
& =2 \operatorname{Re}\left[\mathbf{b}^{H}\left(\widehat{\theta}_{k}\right) \widehat{\mathbf{U}}_{n} \mathbf{d}\left(\widehat{\theta}_{k}\right)\right] \\
& =0,
\end{aligned}
$$

where $\mathbf{d}\left(\widehat{\theta}_{k}\right)=\partial \mathbf{b}\left(\widehat{\theta}_{k}\right) / \partial \widehat{\theta}_{K}$. Taking the first-order Taylor series expansion, then

$$
\begin{aligned}
f^{\prime}\left(\widehat{\theta}_{k}\right) \cong & f^{\prime}\left(\theta_{k}\right)+f^{\prime \prime}\left(\theta_{k}\right)\left(\widehat{\theta}_{k}-\theta_{k}\right) \\
= & 2 \operatorname{Re}\left[\mathbf{b}^{H}\left(\theta_{k}\right) \widehat{\mathbf{U}}_{n} \mathbf{d}\left(\theta_{k}\right)\right] \\
& +2 \operatorname{Re}\left[\mathbf{d}^{H}\left(\theta_{k}\right) \widehat{\mathbf{U}}_{n} \mathbf{d}\left(\theta_{k}\right)\right. \\
& \left.\quad+\mathbf{b}^{H}\left(\theta_{k}\right) \widehat{\mathbf{U}}_{n} \mathbf{d}^{\prime}\left(\theta_{k}\right)\right]\left(\widehat{\theta}_{k}-\theta_{k}\right) \\
\cong & 2 \operatorname{Re}\left[\mathbf{b}^{H}\left(\theta_{k}\right) \widehat{\mathbf{U}}_{n} \mathbf{d}\left(\theta_{k}\right)\right] \\
& +2\left[\mathbf{d}^{H}\left(\theta_{k}\right) \mathbf{U}_{n} \mathbf{d}\left(\theta_{k}\right)\right]\left(\widehat{\theta}_{k}-\theta_{k}\right) \\
= & 0,
\end{aligned}
$$

where some small items are neglected. The mean square error of the angle estimation can be shown as

$$
E\left[\left(\widehat{\theta}_{k}-\theta_{k}\right)^{2}\right]=\frac{\left(\operatorname{Re}\left[\mathbf{b}^{H}\left(\theta_{k}\right) \widehat{\mathbf{U}}_{n} \mathbf{d}\left(\theta_{k}\right)\right]\right)^{2}}{\left(\mathbf{d}^{H}\left(\theta_{k}\right) \mathbf{U}_{n} \mathbf{d}\left(\theta_{k}\right)\right)^{2}} .
$$


According to [28],

$$
\begin{aligned}
\left(\operatorname{Re}\left[\mathbf{b}^{H}\left(\theta_{k}\right) \widehat{\mathbf{U}}_{n} \mathbf{d}\left(\theta_{k}\right)\right]\right)^{2} \\
\quad=\frac{1}{2 J} \mathbf{d}^{H}\left(\theta_{k}\right) \mathbf{U}_{n} \mathbf{d}\left(\theta_{k}\right) \cdot \mathbf{b}^{H}\left(\theta_{k}\right) \mathbf{W}^{1 / 2} \mathbf{U} \mathbf{W}^{1 / 2} \mathbf{b}\left(\theta_{k}\right),
\end{aligned}
$$

where $\mathbf{U}=\mathbf{E}_{s} \mathbf{W}_{s} \mathbf{E}_{s}^{H}$ with $\mathbf{W}_{s}=\operatorname{diag}\left\{\lambda_{1} \sigma^{2} /\left(\lambda_{1}-\sigma^{2}\right)^{2}, \ldots\right.$, $\left.\lambda_{K} \sigma^{2} /\left(\lambda_{K}-\sigma^{2}\right)^{2}\right\}$. Thus the theoretical mean error of the angle estimation of the proposed algorithm can be derived as

$$
E\left[\left(\widehat{\theta}_{k}-\theta_{k}\right)^{2}\right]=\frac{1}{2 J} \frac{\mathbf{b}^{H}\left(\theta_{k}\right) \mathbf{W}^{1 / 2} \mathbf{U} \mathbf{W}^{1 / 2} \mathbf{b}\left(\theta_{k}\right)}{\mathbf{d}^{H}\left(\theta_{k}\right) \mathbf{U}_{n} \mathbf{d}\left(\theta_{k}\right)} .
$$

The theoretical result and the simulation result of the proposed algorithm will be compared in Section 5, where it can be shown that they are almost the same, which demonstrate the correctness of the derivation above.

4.2. Advantages of the Proposed Algorithm. The advantages of the proposed algorithm can be summarized as follows.

(1) It works well with unknown mutual coupling. According to (9), (13), and (14), it can be shown the mutual coupling can be eliminated regardless of the specific distribution of the mutual coupling (the number of the antennas that are influenced by the mutual coupling should be known, i.e., $m$ and $n[21,22])$.

(2) It requires no peak search and has lower complexity, which has been shown in Figure 2.

(3) It has better angle estimation performance than MUSIC-like algorithm and ESPRIT-like algorithm. Part of the reason of this advantage can be refered to "Remark 1", and another reason may be the reduceddimension transformation. According to (13)-(14), the signal to noise ratios (SNRs) of the data before the reduced-dimension transformation and the data after the reduced-dimension transformation are

$$
\begin{aligned}
\mathrm{SNR}_{x c} & =\frac{\operatorname{tr}\left(\mathbf{A}_{1} \mathbf{R}_{s} \mathbf{A}_{1}^{H}\right)}{\operatorname{tr}\left(\mathbf{J}_{1} \otimes \mathbf{J}_{2} E\left[\mathbf{n}(t) \mathbf{n}^{H}(t)\right] \mathbf{J}_{1}^{H} \otimes \mathbf{J}_{2}^{H}\right)} \\
& =\frac{\operatorname{tr}\left(\mathbf{A}_{1} \mathbf{R}_{s} \mathbf{A}_{1}^{H}\right)}{\overline{M N} \sigma^{2}}, \\
\mathrm{SNR}_{y} & =\frac{\operatorname{tr}\left(\mathbf{W}^{1 / 2} \mathbf{B R}_{s} \mathbf{B}^{H} \mathbf{W}^{1 / 2}\right)}{\operatorname{tr}\left(\sigma^{2} \mathbf{I}_{L}\right)}=\frac{\operatorname{tr}\left(\mathbf{G}^{H} \mathbf{A}_{1} \mathbf{R}_{s} \mathbf{B}^{H}\right)}{L \sigma^{2}} \\
& =\frac{\operatorname{tr}\left(\mathbf{A}_{1} \mathbf{R}_{s} \mathbf{A}_{1}^{H}\right)}{(\bar{M}+\bar{N}-1) \sigma^{2}},
\end{aligned}
$$

where $\mathbf{A}_{1}=\left[\mathbf{a}_{r 1}\left(\theta_{1}\right) \otimes \mathbf{a}_{t 1}\left(\theta_{1}\right), \ldots, \mathbf{a}_{r 1}\left(\theta_{K}\right) \otimes \mathbf{a}_{t 1}\left(\theta_{K}\right)\right]$. It can be shown that $\left(\mathrm{SNR}_{y} / \mathrm{SNR}_{x c}\right)=\overline{M N} /(\bar{M}+\bar{N}-1)$, so the reduced-dimension transformation can obtain SNR gain. Advantage 3 can also be verified in Section 5, where the three algorithms are compared.

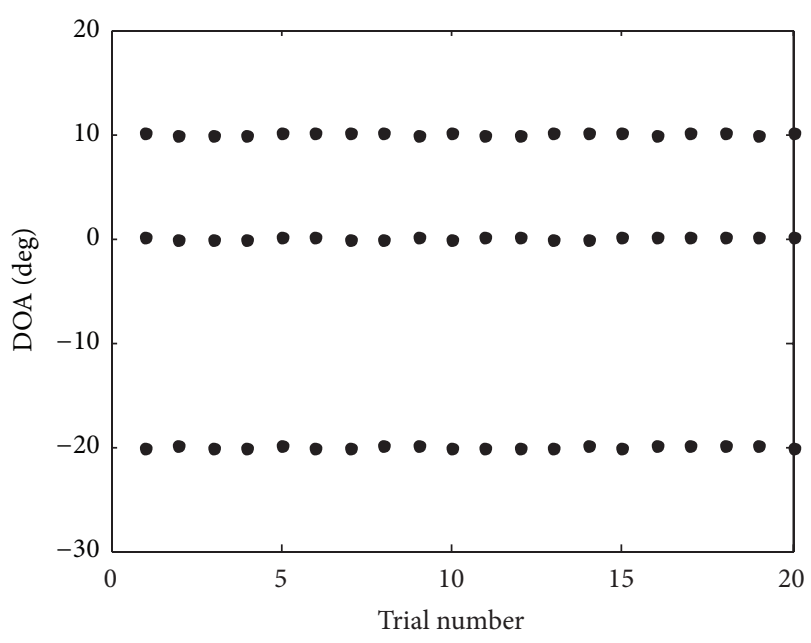

FIGURE 3: Angle estimation result of the proposed algorithm (SNR = $5 \mathrm{~dB})$.

Remark 1. Although MUSIC-like algorithm [24] can achieve high resolution angle estimation with unknown mutual coupling, the peak searches with high complexity will make it ineffective. ESPRIT-like algorithm [25] can obtain the closeform solution of the angle estimation with lower complexity, but it only exploits the relationship between the subarrays, which will lead to the performance loss.

4.3. Cramer-Rao Bound (CRB). According to [29], the CRB for DOA estimation in monostatic MIMO radar with unknown mutual coupling can be derived as

$$
\mathrm{CRB}=\frac{\sigma^{2}}{2}\left\{\operatorname{Re}\left[\mathbf{D}^{H} \boldsymbol{\Pi}_{\mathbf{G}}^{\perp} \mathbf{D}\right]\right\}^{-1},
$$

where $\mathbf{D}=\left[\partial \mathbf{u} / \partial \theta_{1}, \ldots, \partial \mathbf{u} / \partial \theta_{K}, \partial \mathbf{u} / \partial c_{1}^{t}, \ldots, \partial \mathbf{u} / \partial c_{m}^{t}, \partial \mathbf{u} / \partial c_{1}^{r}\right.$, $\left.\ldots, \partial \mathbf{u} / \partial c_{n}^{r}\right]$ with $\mathbf{u}=\left[\begin{array}{c}\widetilde{\mathbf{A}} \mathbf{s}(1) \\ \vdots \\ \widetilde{\mathbf{A} s}(J)\end{array}\right] ; \Pi_{\mathbf{G}}^{\perp}=I_{\mathbf{J}} \otimes \Pi_{\widetilde{\mathbf{A}}}^{\perp}$ with $\Pi_{\widetilde{\mathbf{A}}}^{\perp}=$ $\mathbf{I}_{M N}-\widetilde{\mathbf{A}}\left(\widetilde{\mathbf{A}}^{H} \widetilde{\mathbf{A}}\right)^{-1} \widetilde{\mathbf{A}}^{H}$.

\section{Simulation Results}

Define root mean square error (RMSE) as (1/ K) $\sum_{k=1}^{K} \sqrt{\left((1 / 1000) \sum_{l=1}^{1000}\left[\left(\hat{\theta}_{k, l}-\theta_{k}\right)^{2}\right]\right)}$, where $\widehat{\theta}_{k, l}$ is the estimate of DOA $\theta_{k}$ of the $l$ th Monte Carlo trial. We assume that there are $K=3$ targets with the angles being $\left(\theta_{1}, \theta_{2}, \theta_{3}\right)=\left(-20^{\circ}, 0^{\circ}, 10^{\circ}\right)$. The reflect coefficients are $\left(\beta_{1}\right.$, $\left.\beta_{2}, \beta_{3}\right)=\left[1, e^{j \pi / 5}, 0.8 e^{j \pi / 4}\right]$ and the Doppler frequencies are $\left(f_{1}, f_{2}, f_{3}\right)=[1000 \mathrm{~Hz}, 2550 \mathrm{~Hz}, 5000 \mathrm{~Hz}] / 10^{5}$. The mutual coupling coefficients are $[1,0.1174+0.0577 j]$ and $[1$, $-0.0121-0.1029 j]$ for the transmit and receive arrays, respectively.

Figure 3 depicts angle estimation result of the proposed algorithm with $M=10, N=8, J=100$, and $\mathrm{SNR}=5 \mathrm{~dB}$. It is shown that the DOAs can be clearly observed.

The angle estimation performance comparison between the proposed algorithm, ESPRIT-like algorithm, and 


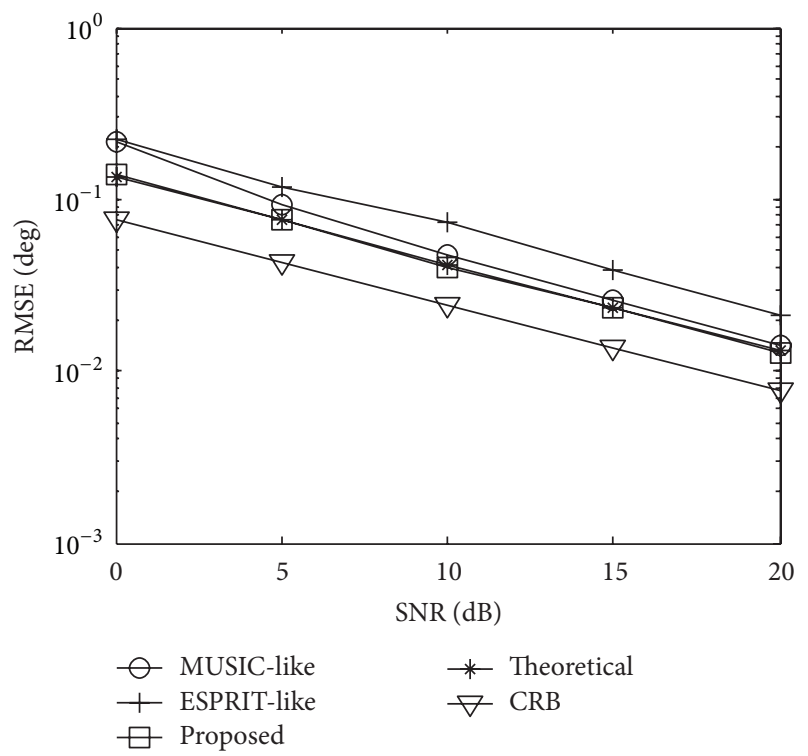

FIGURE 4: Angle estimation performance comparison $(M=10, N=$ $8, J=100)$.

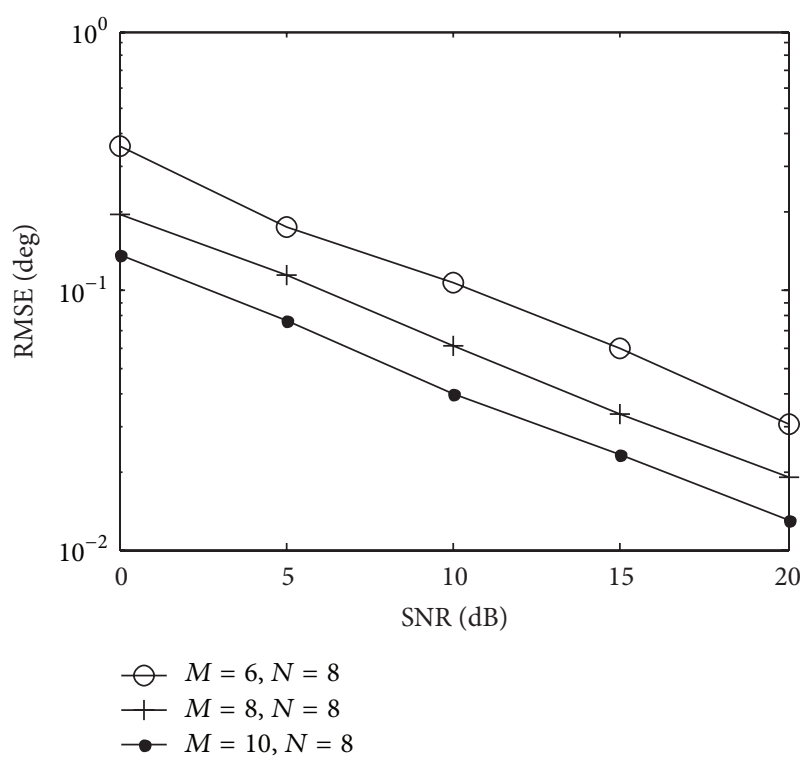

Figure 5: Angle estimation performance with different $M$.

MUSIC-like algorithm is shown in Figure 4, from which we can find that the angle estimation performance of the proposed algorithm is better than that of ESPRIT-like and MUSIC-like. It can also be indicated that the theoretical estimation error and the simulation error of the proposed algorithm are almost the same, which demonstrate the correctness of the derivation in (26).

Figures 5 and 6 present the estimation performance of the proposed algorithm with $J=100$ and variable values of $M$ or $N$. It can be indicated that the angle estimation performance of the proposed algorithm is improved with the increase of receive or transmit antenna numbers because of the diversity gain.

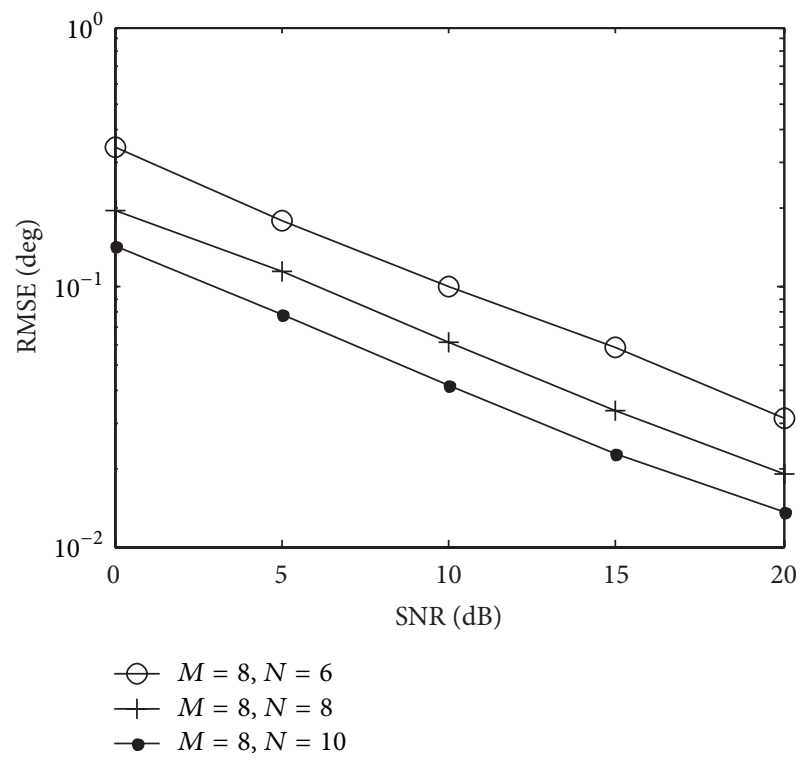

FIGURE 6: Angle estimation performance with different $N$.

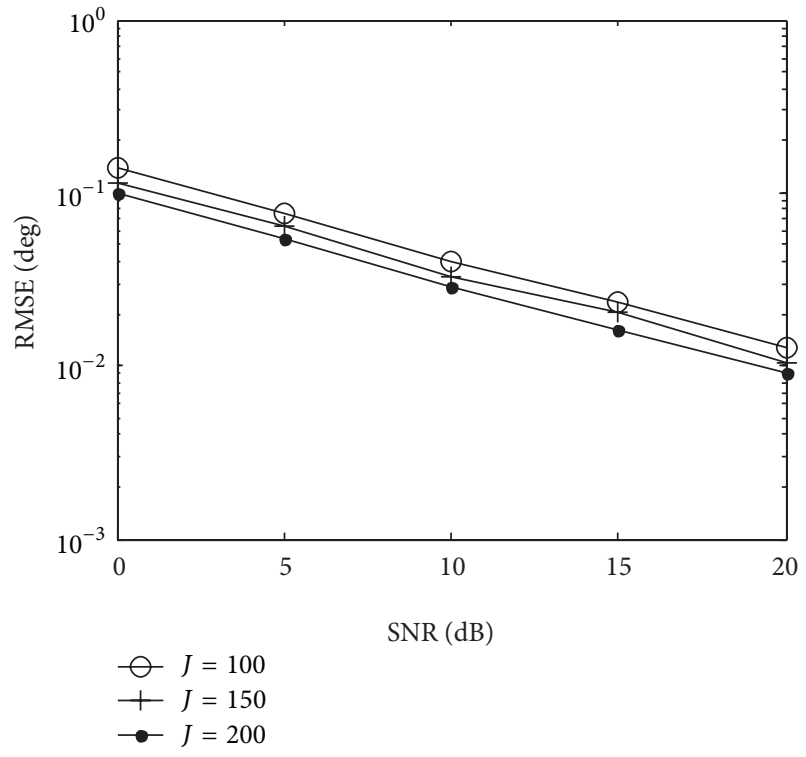

Figure 7: Angle estimation performance with different $J$.

Figure 7 presents the angle estimation performance of our scheme with $M=10, N=8$, and different values of $J$. It has been shown in (26) that the angle estimation error will be reduced when $J$ increases.

To further demonstrate the advantages of the proposed algorithm, another set of parameters is chosen to test the algorithms. Assume that the angles are $\left(\theta_{1}, \theta_{2}, \theta_{3}\right)=\left(-30^{\circ}\right.$, $\left.-20^{\circ}, 10^{\circ}\right)$. The reflect coefficients are $\left(\beta_{1}, \beta_{2}, \beta_{3}\right)=[1$, $\left.0.7 e^{j \pi / 6}, e^{j \pi / 4}\right]$ and the Doppler frequencies are $\left(f_{1}, f_{2}, f_{3}\right)=$ $[2000 \mathrm{~Hz}, 3550 \mathrm{~Hz}, 6000 \mathrm{~Hz}] / 10^{5}$. The mutual coupling coefficients are $[1,0.2134+0.1537 j]$ and $[1,-0.1121-0.1425 j]$ for the transmit and receive arrays, respectively. The RMSE comparison of the algorithms is shown in Figure 8, from 


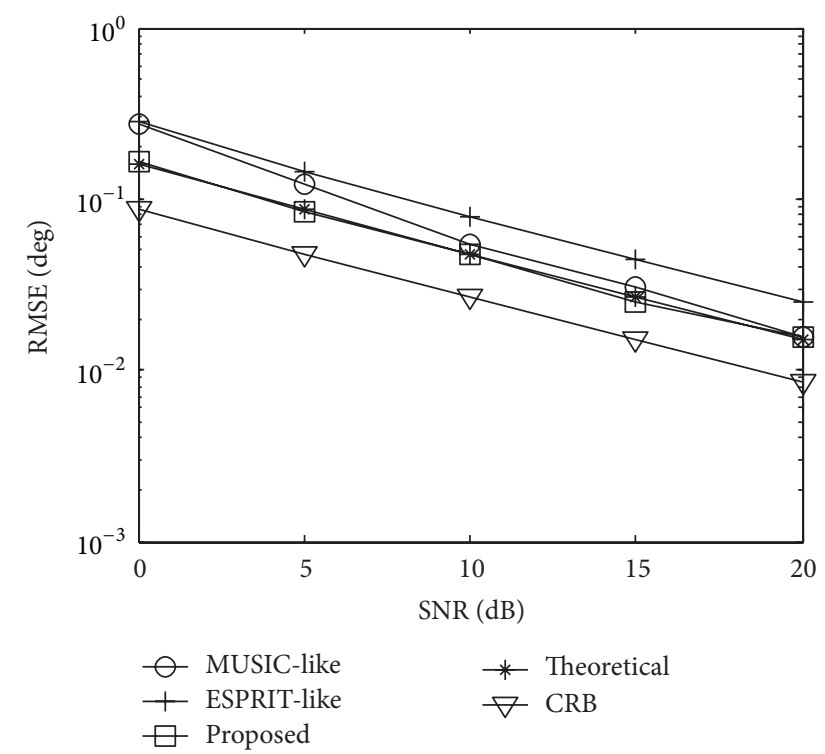

Figure 8: Angle estimation performance comparison (different configuration).

which we can still find that the angle estimation performance of the proposed algorithm is better than the other two algorithms. It can also be indicated that the theoretical estimation error and the simulation error of the proposed algorithm are still the same.

\section{Conclusion}

In this paper, a DOA estimation algorithm based on RootMUSIC has been proposed for monostatic MIMO radar with unknown mutual coupling. The proposed algorithm has low complexity and works fast, requires neither peak searches nor iterations, and can work well with practical unknown mutual coupling. So it can support practical online processing. Furthermore, the proposed algorithm has better angle estimation performance then the ESPRIT-like algorithm and MUSIClike algorithm, which have higher complexity. Many thanks are due to the reviewer's comments; our future work is to test the algorithms with real world data, and try to convert the theories into practical applications.

\section{Conflict of Interests}

The authors declare that there is no conflict of interests regarding the publication of this paper.

\section{Acknowledgments}

This work is supported by the China NSF Grants (61371169, 61071164), the Jiangsu Planned Projects for Postdoctoral Research Funds (1201039C), the China Postdoctoral Science Foundation (2012M521099, 2013M541661), the Open project of key laboratory of underwater acoustic communication and marine information technology (Xiamen University), the Hubei Key Laboratory of Intelligent
Wireless Communications (IWC2012002), the Open project of Key Laboratory of modern acoustic of Ministry of Education (Nanjing University), the Aeronautical Science Foundation of China (20120152001), Funding of Jiangsu Innovation Program for Graduate Education (CXZZ13_0165), Funding for Outstanding Doctoral Dissertation in NUAA (BCXJ13-09), the Qing Lan Project, priority academic program development of Jiangsu high education institutions, and the Fundamental Research Funds for the Central Universities (NS2013024, kfjj130114).

\section{References}

[1] E. Fishler, A. Haimovich, R. Blum, D. Chizhik, L. Cimini, and R. Valenzuela, "MIMO radar: an idea whose time has come," in Proceedings of the IEEE Radar Conference, pp. 71-78, April 2004.

[2] J. Li and P. Stoica, "MIMO radar-diversity means superiority," in Proceedings of the Adaptive Sensor Array Processing Workshop (ASAP '06), pp. 1-6, Lincoln Lab, December 2006.

[3] X.-R. Li, Z. Zhang, W.-X. Mao, X.-M. Wang, J. Lu, and W.-S. Wang, "A study of frequency diversity MIMO radar beamforming," in Proceedings of the IEEE 10th International Conference on Signal Processing (ICSP '10), pp. 352-356, October 2010.

[4] R. Sharma, "Analysis of MIMO radar ambiguity functions and implications on clear region," in Proceedings of the IEEE International Radar Conference (RADAR '10), pp. 544-548, June 2010.

[5] J. Li, G. Liao, and H. Griffiths, "Bistatic MIMO radar spacetime adaptive processing," in Proceedings of the IEEE Radar Conference: In the Eye of the Storm (RadarCon '11), pp. 498-501, Westin Crown Center in Kansas City, May 2011.

[6] X. H. Wu, A. A. Kishk, and A. W. Glisson, "MIMO-OFDM radar for direction estimation," IET Radar, Sonar \& Navigation, vol. 4, no. 1, pp. 28-36, 2010.

[7] J. Li, G. Liao, K. Ma, and C. Zeng, "Waveform decorrelation for multitarget localization in bistatic MIMO radar systems," in Proceedings of the IEEE International Radar Conference (RADAR '10), pp. 21-24, May 2010.

[8] C. Duofang, C. Baixiao, and Q. Guodong, "Angle estimation using ESPRIT in MIMO radar," Electronics Letters, vol. 44, no. 12, pp. 770-771, 2008.

[9] C. Jinli, G. Hong, and S. Weimin, "Angle estimation using ESPRIT without pairing in MIMO radar," Electronics Letters, vol. 44, no. 24, pp. 1422-1423, 2008.

[10] G. Zheng, B. Chen, and M. Yang, "Unitary ESPRIT algorithm for bistatic MIMO radar," Electronics Letters, vol. 48, no. 3, pp. 179-181, 2012.

[11] J. Li, H. Yan, and G. Liao, "Multitarget identification and localization using bistatic MIMO radar systems (EURASIP Journal on Advances in Signal Processing)," Eurasip Journal on Advances in Signal Processing, vol. 2008, Article ID 973932, 2008.

[12] X. Zhang, L. Xu, L. Xu, and D. Xu, "Direction of departure (DOD) and direction of arrival (DOA) estimation in MIMO radar with reduced-dimension MUSIC," IEEE Communications Letters, vol. 14, no. 12, pp. 1161-1163, 2010.

[13] M. L. Bencheikh, Y. Wang, and H. He, "Polynomial root finding technique for joint DOA DOD estimation in bistatic MIMO radar," Signal Processing, vol. 90, no. 9, pp. 2723-2730, 2010.

[14] D. Nion and N. D. Sidiropoulos, "A PARAFAC-based technique for detection and localization of multiple targets in a MIMO 
radar system," in Proceedings of the IEEE International Conference on Acoustics, Speech, and Signal Processing (ICASSP '09), pp. 2077-2080, April 2009.

[15] X. Zhang, Z. Xu, L. Xu, and D. Xu, “Trilinear decompositionbased transmit angle and receive angle estimation for multipleinput multiple-output radar," IET Radar, Sonar and Navigation, vol. 5, no. 6, pp. 626-631, 2011.

[16] D. Nion and N. D. Sidiropoulos, "Adaptive algorithms to track the PARAFAC decomposition of a third-order tensor," IEEE Transactions on Signal Processing, vol. 57, no. 6, pp. 2299-2310, 2009.

[17] X. Zhang and D. Xu, "Low-complexity ESPRIT-based DOA estimation for colocated MIMO radar using reduced-dimension transformation," Electronics Letters, vol. 47, no. 4, pp. 283-284, 2011.

[18] X. Zhang, Y. Huang, C. Chen et al., "Reduced-complexity Capon for direction of arrival estimation in a monostatic multiple-input multiple-output radar," IET Radar, Sonar \& Navigation, vol. 6, no. 8, pp. 796-801, 2012.

[19] H.-S. Lui, H. T. Hui, and M. S. Leong, "A note on the mutualcoupling problems in transmitting and receiving antenna arrays," IEEE Antennas and Propagation Magazine, vol. 51, no. 5, pp. 171-176, 2009.

[20] H. S. Lui and H. T. Hui, "Mutual coupling compensation for direction-of-arrival estimations using the receiving-mutualimpedance method," International Journal of Antennas and Propagation, vol. 2010, Article ID 373061, 7 pages, 2010.

[21] J. Dai, D. Zhao, and X. Ji, "A sparse representation method for DOA estimation with unknown mutual coupling," IEEE Antennas and Wireless Propagation Letters, vol.11, pp. 1210-1213, 2012.

[22] Z. Ye, J. Dai, X. Xu, and X. Wu, "DOA estimation for uniform linear array with mutual coupling," IEEE Transactions on Aerospace and Electronic Systems, vol. 45, no. 1, pp. 280-288, 2009.

[23] J. Li and X. Zhang, "Joint angles and mutual coupling estimation algorithm for bistatic MIMO radar," International Journal of Antennas and Propagation, vol. 2012, Article ID 921878, 8 pages, 2012.

[24] X. Liu and G. Liao, "Direction finding and mutual coupling estimation for bistatic MIMO radar," Signal Processing, vol. 92, no. 2, pp. 517-522, 2012.

[25] Z. D. Zheng, J. Zhang, and J. Y. Zhang, "Joint DOD and DOA estimation of bistatic MIMO radar in the presence of unknown mutual coupling," Signal Processing, vol. 92, no. 12, pp. 30393048, 2012.

[26] P. Chargé, Y. Wang, and J. Saillard, "A non-circular sources direction finding method using polynomial rooting," Signal Processing, vol. 81, no. 8, pp. 1765-1770, 2001.

[27] B. D. Rao and K. V. S. Hari, "Performance analysis of RootMusic," IEEE Transactions on Acoustics, Speech, and Signal Processing, vol. 37, no. 12, pp. 1939-1949, 1989.

[28] P. Stoica and A. Nehorai, "MUSIC, maximum likelihood, and Cramer-Rao bound," IEEE Transactions on Acoustics, Speech, and Signal Processing, vol. 37, no. 5, pp. 720-741, 1989.

[29] P. Stoica and A. Nehorai, "Performance study of conditional and unconditional direction-of-arrival estimation," IEEE Transactions on Acoustics, Speech, and Signal Processing, vol. 38, no. 10, pp. 1783-1795, 1990. 

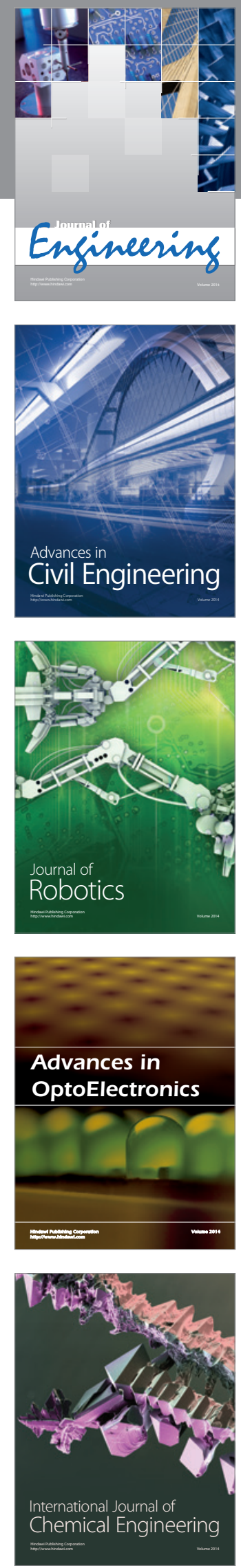

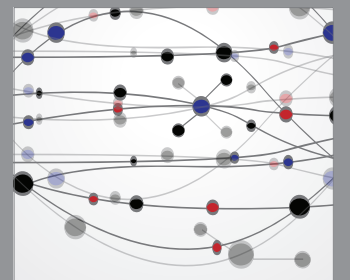

The Scientific World Journal
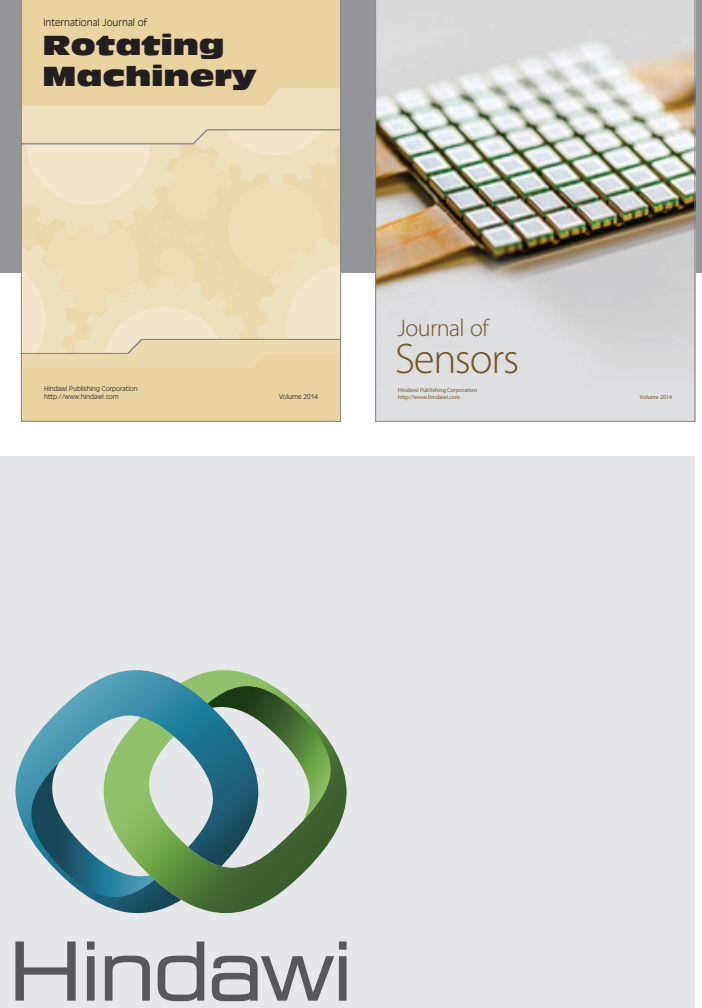

Submit your manuscripts at http://www.hindawi.com
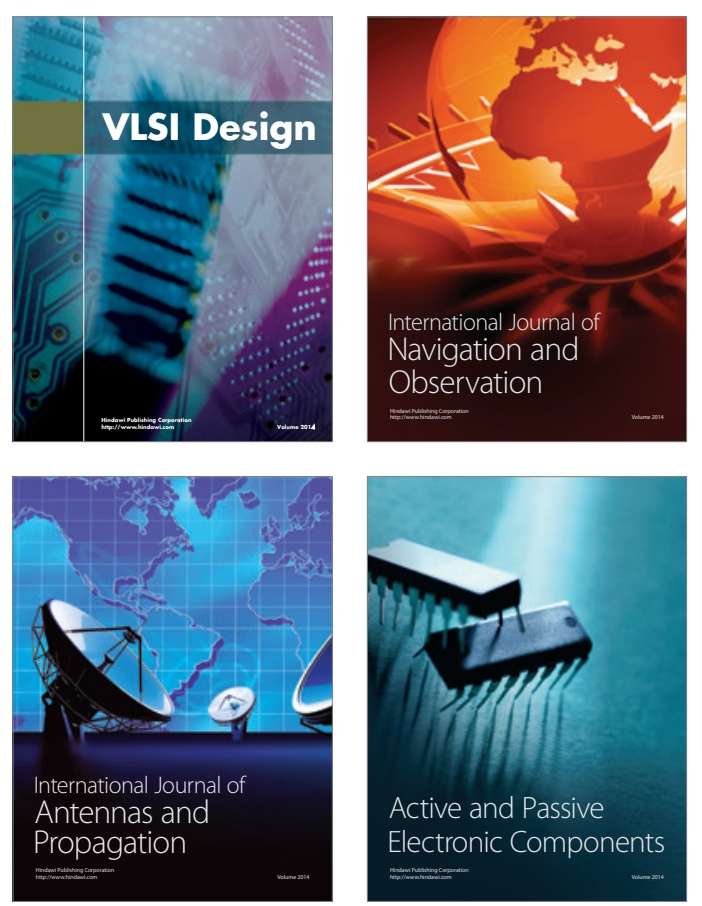
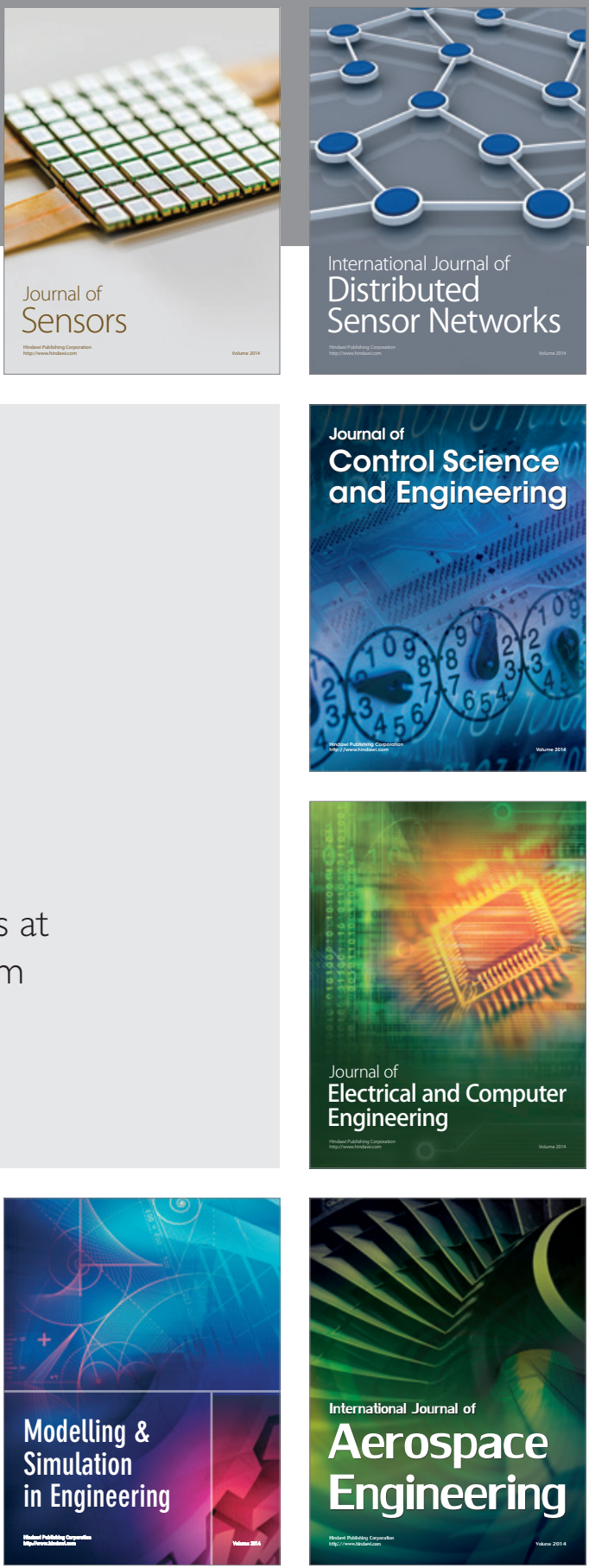

Journal of

Control Science

and Engineering
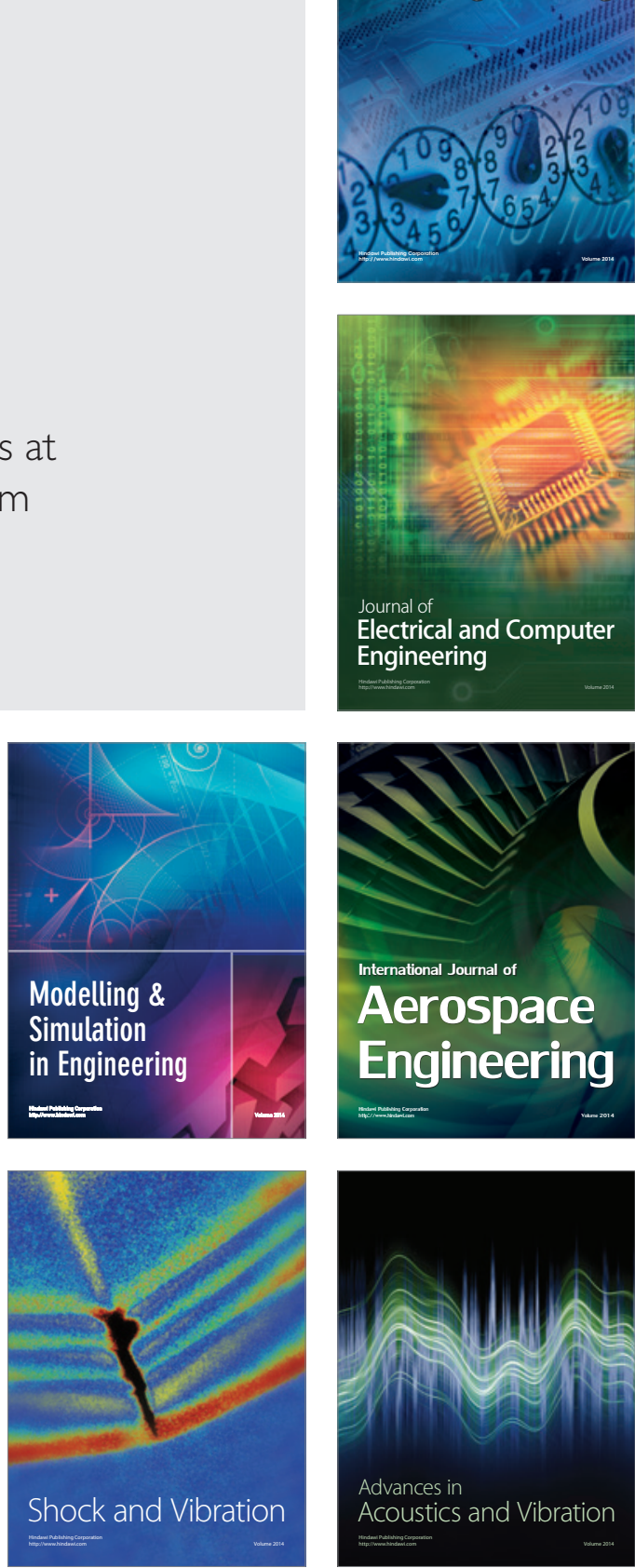